\title{
Research on Remote Sensing Hazard Clouds Basing on Continuous Point Source
}

\author{
Shunhua Liu ${ }^{1}$, Xuezheng Zhu ${ }^{1}$, Zhirong Luo ${ }^{1}$, Kunlin Nie ${ }^{1}$, Xiaobin Liu ${ }^{2}$ \\ ${ }^{1}$ Institute of Chemical Defense \\ ${ }^{2}$ Unit 31679 \\ 18610460988@wo.com.cn
}

Keywords: continuous point source; hazard clouds; remote sensing; modeling

Abstract: Employing infrared telemetry principle and diffusion theory, this paper has established remote sensing model of continuous point source hazard clouds according to its density, harm in-depth and corner relationship. After a series of experiments, this model is convincible in effectively realize its immediate positioning and hazard evaluation matters with little mistakes.

\section{Introduction}

The chemical weapon usage in battle-period and its pollution air from chemical plants are exerting long lasting and wide spread pollution around due to the fact that environment pollution has appeared with an increasing tendency in recent years, which calls for a closer monitoring management. Remote sensing Fourier transform infrared (RS-FTIR), as an effective monitoring method, can further detect the pollution air several kilometers away and is increasingly employed in the pollution air detection ${ }^{[1]}$. The cloud cluster detection is rather hard to realize for the hazard clouds happens out of no-where, no-when and no-how. While the continuous point source happens in a detectable mode in both daily and battle time, this paper mainly focuses on the telemetry positioning research of continuous point source hazard clouds.

\section{Proposition}

The shape of hazard clouds is closely related with the release sources that can be divided into four types of point, line, non-point and volume source ${ }^{[2]}$. There are transient point-source and continuous point-source. Continuous point-source refers to the ones continuously released such as smokestack, chemical reaction kettle and smoke can. Hazard clouds out of continuous point source will quickly diffuse into the leeward direction. When its pollution density reaches the limit value, this hazard clouds will shape itself into a balanced circumstance with a stable hazardous depth that normally depends on the current wind speed and point source release rate. There comes the difficulty of continuous point source hazard clouds positioning because of the not detectable character, changeable weather condition and influential hazardous depth.

With the infrared telemetry technology, the detection of hazard cloudss is shown as the following Figure1 illustrating the received radiance ${ }^{[3]}$.

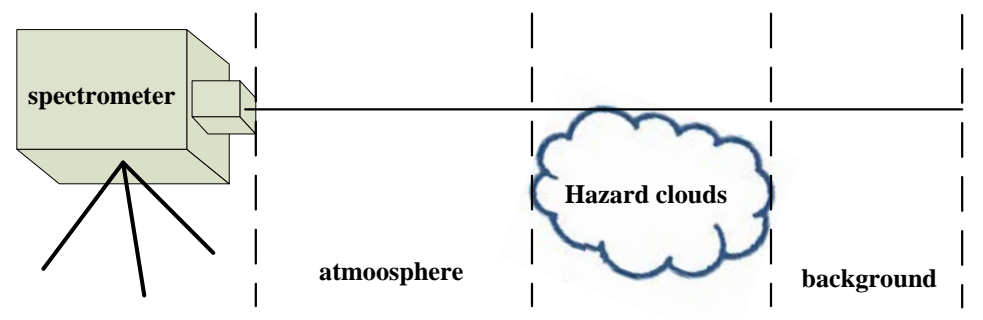

Figure 1 infrared telemetry principle

According to the Planck's radiation law, the radiance is changed as the brightness temperature. Then a combined analysis is conducted of far-infrared spectra collection, brightness temperature 
spectra feature basing on FTIR analyzing of hazard clouds and the brightness temperature difference between the facial and telemetry layer. Finally the concentration range product of the hazard clouds can be concluded ${ }^{[4]}$. When this value reaches its limitation, it will automatically trigger the alarm system. Concluding from the working mechanism, this infrared telemetry system can detect the target's azimuth and position coordinates, but still not enough to the exact location.

Thus there still needs further work on how to precisely detect the location no matter it's in battle or daily chemical protection to offer accurate and real-time information for the commanders.

\section{Model Establishment}

\subsection{Conditional assumption}

Infrared telemetering device can determine its own position through the Beidou or GPS positioning module, and then 360-degree global scan monitoring. If at time $t_{0}$, the infrared telemetering device scans the polluted cloud and the direction angle of the scan at time $t_{0}$ is $\alpha$, It is assumed that the point of tangency of ray along the direction angle and the boundary point of the pollution cloud is $A$, (The concentration line formed by infrared remote sensing alarm concentration threshold is set to the boundary line of pollution cloud). According to Figure 2, the relevant circumstances are assumed:

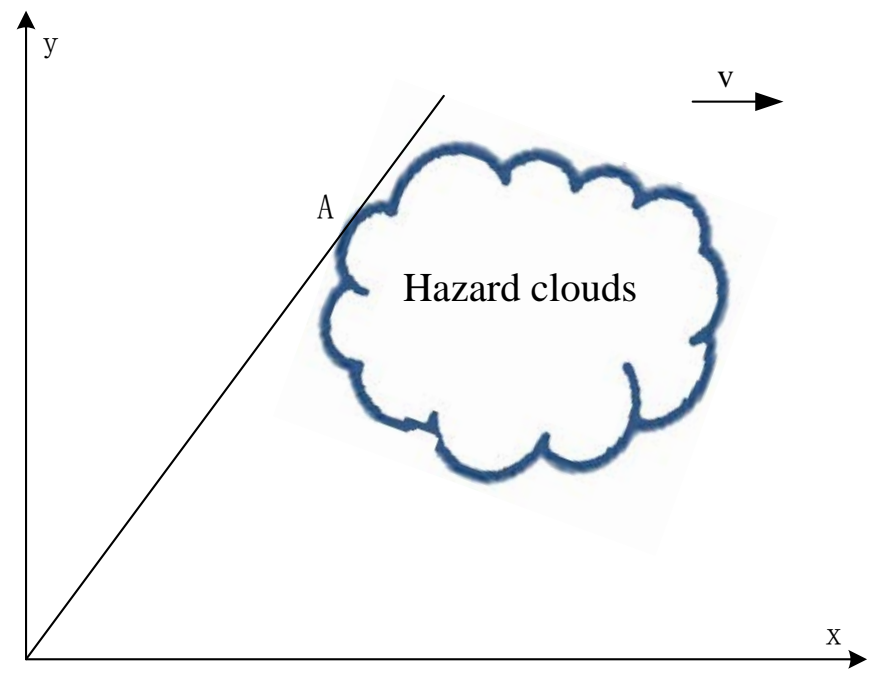

Figure 2 schematic diagram of scanning infrared monitoring device for monitoring hazard clouds

(1) Focusing on the relationship between the location of the cloud and damage areas, the coordinates can be simplified into two-dimensional plane space, infrared telemetering device for the plane coordinates of a monitoring point;

(2) Infrared telemetering device for the pollution cloud alarm alarm threshold is $C_{0}$, According to the turbulence diffusion theory, the concentration of cloud at any point varies with time, and the concentration of the pollution cloud is $C_{0}$;

(3) The meteorological conditions are constant, the underlying surface is flat and open, The axial diffusion coefficients $\left(K_{0}\right)$ of polluted cloud $\mathrm{X}$ axis and $\mathrm{Y}$ axis are equal;

(4) The continuous point source releases the chemical substances and reaches a balance, i.e. damage to the depth and remains relatively fixed;

(5) It is assumed that wind speed is stable, the wind direction does not change, and is parallel to the $x$ axis, the wind speed is $u$.

\subsection{Model deduction}

Two points in plane coordinates $\mathrm{A}_{0}\left(\mathrm{x}_{\mathrm{A} 0}, \mathrm{y}_{\mathrm{A} 0}\right) 、 B_{0}\left(\mathrm{x}_{\mathrm{B} 0}, \mathrm{y}_{\mathrm{B} 0}\right)$, and each point represents an infrared telemetry device. At the same time, the 360 degree global scan monitors the pollution cloud. When the alarm occurs, the direction angle is recorded automatically by the direction finder as 
$\theta_{A} 、 \theta_{B}$, as shown in Figure 3.

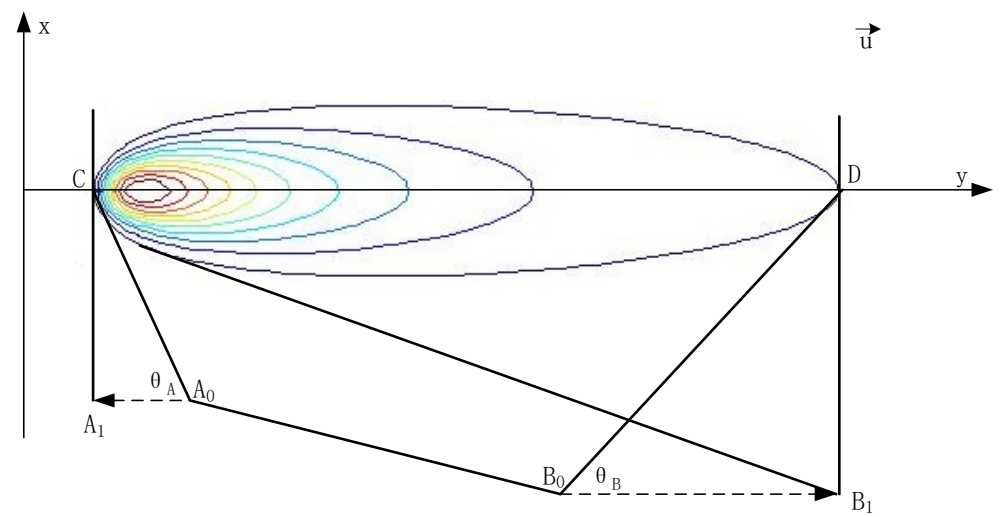

Figure 3 schematic diagram of telemetry positioning of continuous point source hazard clouds

Two sets of infrared telemetry devices are maneuvering along the wind direction, make $\theta_{\mathrm{A}} 、 \theta_{\mathrm{B}}$ change to $90^{\circ}$, Record the position coordinates of the infrared telemetering device when $\theta_{\mathrm{A}}=90^{\circ} 、 \theta_{\mathrm{B}}=90^{\circ}$, mark as the point, calculate the length of the line $A_{0} A_{1} 、 B_{0} B_{1}$.

$$
A_{0} A_{1}=\left|y_{A 1}-y_{A 0}\right| ; \quad B_{0} B_{1}=\left|y_{B 1}-y_{B 0}\right|
$$

As shown in Figure $3 \Delta \mathrm{CA}_{0} \mathrm{~A}_{1} 、 \triangle \mathrm{DB}_{0} \mathrm{~B}_{1}$, Calculate the length of $\mathrm{CA}_{1}$ and $\mathrm{DB}_{1}$ lines according to the trigonometric relation.

$$
C A_{1}=A_{0} A_{1} \times \tan \theta_{A} ; \quad D B_{1}=B_{0} B_{1} \times \tan \theta_{B}
$$

And then according to the coordinate translation, calculate the coordinates of the pollute cloud endpoints $C\left(\mathrm{x}_{A 1}+C A_{1}, \mathrm{y}_{A 1}\right), D\left(\mathrm{x}_{B 1}+D B_{1}, \mathrm{y}_{B 1}\right)$.

According to the theory of turbulence diffusion gradient, we derive the differential equations of Lahtman's chemical cloud propagation:

$$
\frac{\partial C}{\partial t}+u \frac{\partial C}{\partial x}=K_{0}\left(\frac{\partial^{2} C}{\partial x^{2}}+\frac{\partial^{2} C}{\partial y^{2}}\right)+\frac{\partial}{\partial z}\left[K_{1}\left(\frac{z}{Z_{1}}\right)^{2-n} \frac{\partial C}{\partial z}\right]
$$

Model setting condition

- No mass exchange and loss of toxicant with the underlying surface

- Before the chemical attack, the polluted cloud concentration of the space at any point is 0 .

- After the chemical attack, the infinite of pollution cloud concentration is 0 .

- The quality $Q$ of the poison entering the space is conserved

The instantaneous point source is used as the object of study to solve the equation of concentration $^{[5]}$ :

$$
C(\mathrm{x}, \mathrm{y}, \mathrm{z})=\frac{Q}{4 \pi K_{0} t\left(K_{1} n^{2} z_{1}^{n-2} t\right)^{1 / n} \Gamma(1+1 / n)} e^{\left(-\frac{x^{2}+y^{2}}{4 K_{0} t}-\frac{z^{n}}{K_{1} n^{2} z_{1}^{n-2} t}\right)}
$$

$C(\mathrm{x}, \mathrm{y}, \mathrm{z})$ Concentration of poison at some point in space $(\mathrm{x}, \mathrm{y}, \mathrm{z})$ at $t$

$Q$ - The amount of poison entering the air from an instantaneous point source, $\mathrm{g}$;

$u$ - The average speed of the wind, $\mathrm{m} / \mathrm{s}$;

$K_{0}$ _ Turbulence level diffusion coefficient, $\mathrm{m}^{2} / \mathrm{s}$;

$\mathrm{Z}_{1}$ —Fixed reference height,m;

$K_{1}-$ Vertical diffusivity of turbulence at $\mathrm{Z}_{1}$ high altitude, $\mathrm{m}^{2} / \mathrm{s}$;

$n=1-\frac{\Delta t}{u_{1}^{2}} \quad$ Characteristic coefficients of atmospheric vertical stability 
$\Gamma(1+1 / n)$ is the gamma function that varies with $n$.

Continuous point source can be seen as a number of instantaneous point source in chronological order, the formation of pollution cloud propagation direction depends on the wind direction; the speed depends on the wind speed and atmospheric turbulence; and the concentration increases with the increase of the source intensity. Turbulence and the distance increases.

The releasing rate of a continuous point source is $Q_{\mathrm{p}}$, Instantaneous release capacity $Q_{\mathrm{p}} d \mathrm{t}=$ $Q_{\mathrm{p}} d\left(\frac{\mathrm{x}}{u}\right)$, and in the formula (2), for $\mathrm{X}$ integral, there are:

$$
C(\mathrm{x}, \mathrm{y}, \mathrm{z}, \mathrm{t})=\frac{Q_{\mathrm{p}}}{4 \pi K_{0} t\left(K_{1} n^{2} z_{1}^{n-2} t\right)^{1 / n} \Gamma(1+1 / n)} e^{\left(-\frac{y^{2}}{4 K_{0} t}-\frac{z^{n}}{K_{1} n^{2} z_{1}^{n-2} t}\right)} \int_{-\infty}^{+\infty} e^{\left(-\frac{x^{2}}{4 K_{0} t}\right)} d x
$$

Let $\frac{x^{2}}{4 K_{0} t}=\omega^{2}$, then $d x=\sqrt{4 k_{0} t} d \omega$

$$
C(\mathrm{x}, \mathrm{y}, \mathrm{z}, \mathrm{t})=\frac{Q_{\mathrm{p}}}{4 \pi K_{0} t\left(K_{1} n^{2} z_{1}^{n-2} t\right)^{1 / n \Gamma(1+1 / n)}} e^{\left(-\frac{y^{2}}{4 K_{0} t}-\frac{z^{n}}{K_{1} n^{2} z_{1}^{n-2} t}\right)} \int_{-\infty}^{+\infty} e^{\left(-\omega^{2}\right)} d \omega
$$

owing to:

$$
\begin{gathered}
\int_{-\infty}^{+\infty} e^{\left(-\omega^{2}\right)} d \omega=2 \int_{0}^{+\infty} e^{\left(-\omega^{2}\right)} d \omega=\sqrt{\pi} \\
C(\mathrm{x}, \mathrm{y}, \mathrm{z}, \mathrm{t})=\frac{Q_{\mathrm{p}} \sqrt{4 k_{0} t \pi}}{4 \pi K_{0} t\left(K_{1} n^{2} z_{1}^{n-2} t\right)^{1 / n} \Gamma(1+1 / n)} e^{\left(-\frac{y^{2}}{4 K_{0} t}-\frac{z^{n}}{K_{1} n^{2} z_{1}^{n-2} t}\right)} \\
t=x / u \\
C(\mathrm{x}, \mathrm{y}, \mathrm{z})=\frac{Q_{\mathrm{p}}}{2 \sqrt{k_{0} \pi x / u}\left(K_{1} n^{2} z_{1}^{n-2} x / u\right)^{1 / n} \Gamma(1+1 / n)} e^{-\frac{u}{4 x}\left(\frac{y^{2}}{K_{0}}+\frac{4 z^{n}}{K_{1} n^{2} z_{1}^{n-2}}\right)}
\end{gathered}
$$

From the formula (5), In steady state conditions, the concentration of contaminated clouds which produced by continuous point sources having nothing to do with time. In order to facilitate the use of calculation, the formula (5) is simplified, and $z=0 、 z_{1}=1 、 \mathrm{n}=1 、 \Gamma(1+1 / n)=1$, so that there are:

$$
C(x, y)=\frac{Q_{\mathrm{p}}}{2 K_{1} \sqrt{k_{0} \pi}(x / u)^{3 / 2}} e^{-\frac{u y^{2}}{4 K_{0} x}}
$$

Observe the concentration of the chemical cloud end point $D$, then the formula (6) is simplified to:

$$
C_{0}=\frac{Q_{\mathrm{p}}}{2 K_{1} \sqrt{k_{0} \pi}(x / u)^{3 / 2}}
$$

As can be seen from formula (7), polluted cloud trace area produced by continuous point source depth $x$ (ie, depth of danger) are related to wind speed and the amount of discharge capacity, therefore, we can study the infrared telemetering device how to monitor continuous point source. As can be seen from formula (7), If the infrared telemetering device can measure the depth of hazard clouds, that is, the length of the cloud along the wind direction, we can solve the source value $Q_{p}$ of continuous point source, by substituting the value $Q_{p}$ 、x into the formula (6), the coordinates $(\mathrm{x}, \mathrm{y})$ of the alarm concentration threshold $\mathrm{C}_{0}$ can be solved, and the boundary of cloud sources with continuous point source pollution can be determined.

\subsection{Telemetry positioning model}

The establishing equation is as below. 


$$
\left\{\begin{array}{c}
C_{0}=\frac{Q_{\mathrm{p}}}{2 K_{1} \sqrt{k_{0} \pi}(x / u)^{3 / 2}} e^{-\frac{u y^{2}}{4 K_{0} x}} \\
C_{0}=\frac{Q_{\mathrm{p}}}{2 K_{1} \sqrt{k_{0} \pi}(x / u)^{3 / 2}} \\
x=\left|y_{B 1}-y_{A 1}\right|
\end{array}\right.
$$

\section{Model Solution Demonstration}

The two set infrared telemetry device on the facial layer decides their position coordinates as $\mathrm{A}_{0}\left(\mathrm{x}_{\mathrm{A} 0}, \mathrm{y}_{\mathrm{A} 0}\right)=(-600,600), \mathrm{B}_{0}\left(\mathrm{x}_{\mathrm{B} 0}, \mathrm{y}_{\mathrm{B} 0}\right)=(-700,800)$.

Record the meteorological parameters like temperature. Conduct the horizontal diffusion coefficient $\mathrm{K}_{0}$ and vertical diffusion coefficient $\mathrm{K}_{1}$ basing on the meteorological parameter. Suppose the values are as follows: $K_{0}=0.785 \mathrm{~m}^{2} / \mathrm{s}, K_{1}=\frac{0.645 \mathrm{~m}^{2}}{\mathrm{~s}}, u=2 \mathrm{~m} / \mathrm{s}$.

Mark at the point $\left(\mathrm{A}_{0}, \mathrm{~B}_{0}\right)$ according to the wind direction and scan the entire area to monitor the hazard clouds in the meantime. When the alarm system is triggered, the goniometer will record the azimuth and finally converses into $\theta_{A}=60^{\circ} 、 \theta_{B}=45^{\circ}$. Basing on the illustrated poison type on the alarm system, the target's alarm density limitation value can be calculated and recorded as $C_{0}=0.3 \mathrm{~g} / \mathrm{m}^{2}$.

Remove the infrared telemetry device follow the wind making $\theta_{\mathrm{A}} 、 \theta_{\mathrm{B}}$ turn to its $90^{\circ}$ angle and the location coordinates are $A_{1}\left(\mathrm{x}_{A 1}, \mathrm{y}_{A 1}\right)=(-600,311), B_{1}\left(\mathrm{x}_{B 1}, \mathrm{y}_{B 1}\right)=(-700,1400)$. Then there is the length of line $A_{0} A_{1}$ and $B_{0} B_{1}$.

$$
\begin{gathered}
A_{0} A_{1}=\left|y_{A 1}-y_{A 0}\right|=|311-600|=289 \mathrm{~m} \\
B_{0} B_{1}=\left|y_{B 1}-y_{B 0}\right|=|1400-800|=600 \mathrm{~m}
\end{gathered}
$$

In the following $\triangle \mathrm{CA}_{0} \mathrm{~A}_{1} 、 \triangle \mathrm{DB}_{0} \mathrm{~B}_{1}$ in Figure 4, line $C A_{1} 、 D B_{1}$ can be calculated as below.

$$
\begin{aligned}
& C A_{1}=289 \times \tan 60^{\circ}=500 \mathrm{~m} \\
& D B_{1}=600 \times \tan 45^{\circ}=600 \mathrm{~m}
\end{aligned}
$$

Then with the coordinates translation, the end of the hazard clouds is $C(-100,311), D(-100,800) . S=800-311=489 \mathrm{~m}$.

With the continuous point source density equation, the density of end $\mathrm{D}$ is:

$$
C_{0}=\frac{Q_{\mathrm{p}}}{2 K_{1} \sqrt{k_{0} \pi}(S / u)^{3 / 2}}
$$

With the target's hazardous depth (i.e. $S=C D$ length) and average speed wind $u$ putting back into the mentioned equation, the continuous point source release volume $Q_{\mathrm{p}}$ can be calculated as $Q_{\mathrm{p}}=2323.5 \mathrm{~g} / \mathrm{s}$.

With the value $Q_{p}, u$ substituting into equation (6), the alarm density limitation $\mathrm{C}_{0}(\mathrm{x}, \mathrm{y})$ can be calculated to further decide the exact boundary. Figure 4 shows this whole process.

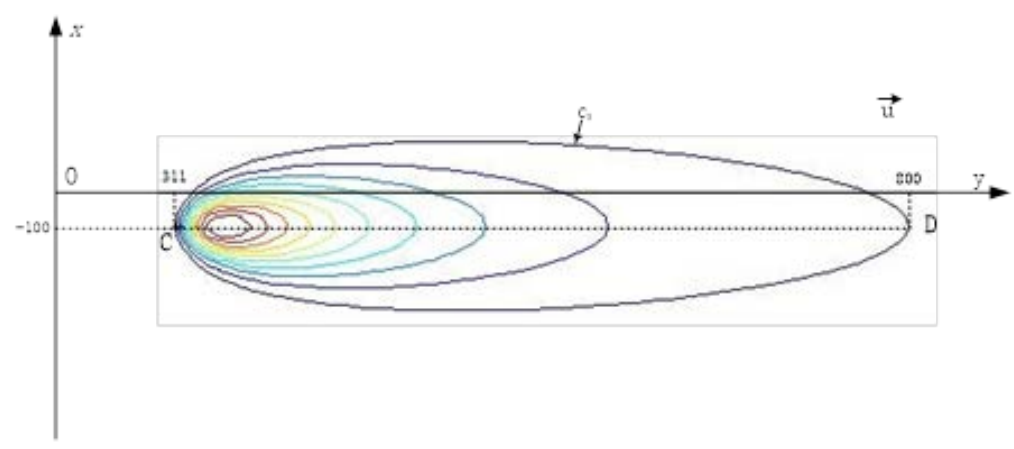

Figure 4 estimating continuous point source hazard clouds positioning and hazardous range 


\section{Analysis and Discussion}

Under the parameter value in equation (8) and formula (7), Figure 5 illustrates the relation between the hazardous depth and its release volume at the balance position in different wind speed.

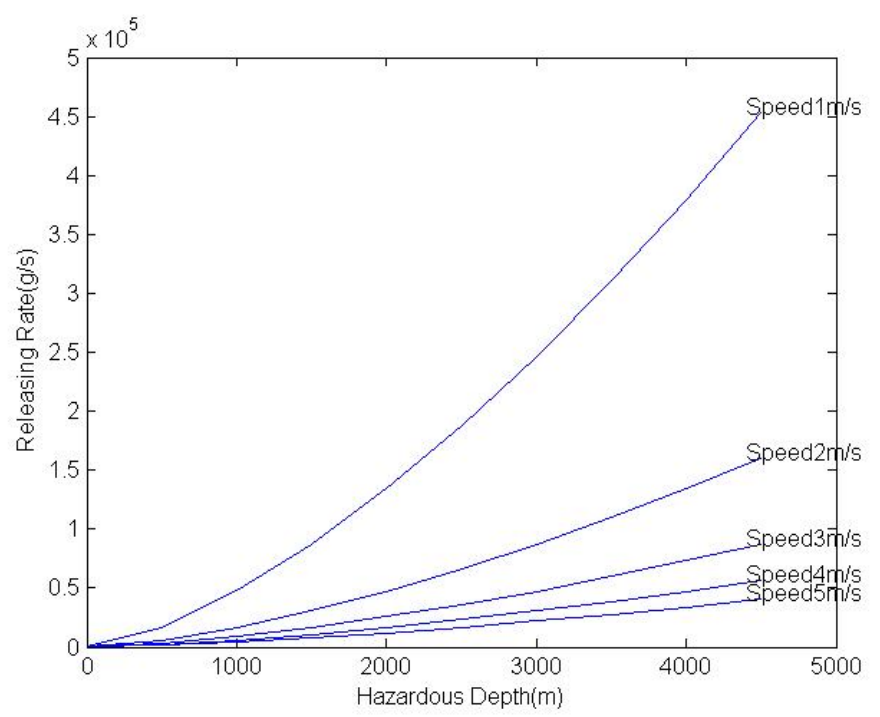

Figure 5 releasing rate and hazardous depth relation under different wind speed of continuous point source in balanced position

Englightened by Figure 5, continuous point source in the balanced situation shows a close relationship among release volume, hazardous depth and wind speed. In the same release volume condition, the hazard clouds's hazardous depth increases with the wind speed; in the same wind speed, the hazardous depth increases with the release volume; in the same hazardous depth, the release volume increases with the wind speed.

Infrared telemetry device mainly focuses on the position coordinates and telemetry angle of continuous hazard clouds. Combining with the mentioned modeling principle, this telemetry model is used to inverse calculate the position and source point by monitoring the hazard clouds's hazardous depth in the balanced circumstance. Given this, there are three notes to be noticed in the telemetry process:

(1) Make sure there are at least two infrared telemetry device working at the same time;

(2) Main telemetry data: position coordinates, telemetry angle;

(3) Keep in the same direction with the wind when moves the infrared telemetry device. If not, please timely update. In order to decrease the location mistake, the telemetry angle $\theta_{A} 、 \theta_{B}$ should bigger than $30^{\circ}$.

\section{References}

[1] Wei Xiong, Yonghua Fang, Huang Ye. Pollution Air Telemetry Detection in the Changeable Background. Laser \& Infrared. Vol.36, No. 5,2006:361-364

[2] HE HUA SHENG FANGHU DA CIDIAN [M]. Shanghai: Shanghai Lexicographical Publishing House,1999:142-143

[3] Wei Xiong, Yonghua Fang, Ye Huang. Pollution Air Telemetry Detection Calculation in a Changeable Background, Infrared, Vol .27, No. 2,2006:6-8

[4] Fangxiao Cui, Yonghua Fang. Infrared Background Compression Method Basing on the Brightness Temperature Spectrum, Acta Optica Sinica, Vol.33, No. 11:1-6

[5] Shunxiang Huang, Haiping Chen. Chemical Risk Evaluation [M]. Beijing: China Meteorological Press. 2010.12 